Revue internationale P.M.E.

Économie et gestion de la petite et moyenne entreprise

\title{
Décision d'investissement des petites entreprises chinoises dans un système de responsabilité
}

\section{Hua $\mathrm{Yu}$}

Volume 6, numéro 1, 1993

URI : https://id.erudit.org/iderudit/1008161ar

DOI : https://doi.org/10.7202/1008161ar

Aller au sommaire du numéro

Éditeur(s)

Presses de l’Université du Québec

ISSN

0776-5436 (imprimé)

1918-9699 (numérique)

Découvrir la revue

Citer cet article

Yu, H. (1993). Décision d'investissement des petites entreprises chinoises dans un système de responsabilité. Revue internationale P.M.E., 6(1), 11-27.

https://doi.org/10.7202/1008161ar

\section{Résumé de l'article}

Dans cet article, l'auteur analyse la nature et les impacts du contrat de location des petites entreprises chinoises, dans un contexte de système de responsabilité. Dans un contrat typique, le locateur du bien convoité est le propriétaire, tandis que le locataire est le gestionnaire qui ne détient qu'une responsabilité partielle, soit celle de verser un montant fixe au locataire à certaines conditions. Il montre que l'entrepreneur-locateur peut accroître sa richesse en augmentant le risque total de l'entreprise louée, lorsque l'information concernant ces décisions extérieures n'est pas totalement divulguée sur le marché. De plus, i'entrepreneur-locataire n'a aucune incitation à réinvestir ses bénéfices dans l'entreprise, étant donné l'incertitude entourant le bénéfice cible indiqué dans le contrat renouvelé. Ces deux aspects peuvent avoir des conséquences importantes sur le développement des entreprises rurales à long terme.
Ce document est protégé par la loi sur le droit d'auteur. L'utilisation des services d’Érudit (y compris la reproduction) est assujettie à sa politique d'utilisation que vous pouvez consulter en ligne.

https://apropos.erudit.org/fr/usagers/politique-dutilisation/ 


\title{
Décision d'investissement des petites entreprises chinoises dans un système de responsabilité
}

\author{
Hua YU* \\ Université du Québec à Trois-Rivières
}

\begin{abstract}
RÉSUMÉ
Dans cet article, l'auteur analyse la nature et les impacts du contrat de location des petites entreprises chinoises, dans un contexte de système de responsabilité. Dans un contrat typique, le locateur du bien convoité est le propriétaire, tandis que le locataire est le gestionnaire qui ne détient qu'une responsabilité partielle, soit celle de verser un montant fixe au locataire à certaines conditions. II montre que l'entrepreneur-locateur peut accroître sa richesse en augmentant le risque total de l'entreprise louée, lorsque l'information concernant ces décisions extérieures n'est pas totalement divulguée sur le marché. De plus, l'entrepreneur-locataire n'a aucune incitation à réinvestir ses bénéfices dans l'entreprise, étant donné l'incertitude entourant le bénéfice cible indiqué dans le contrat renouvelé. Ces deux aspects peuvent avoir des conséquences importantes sur le développement des entreprises rurales à long terme.
\end{abstract}

\begin{abstract}
This paper analyzes the basic characteristics of a lease contract of small firms under the responsibility system in China. In a typical contract, the lessor is the owner of the leased firm, while the lessee is an entrepreneur who has a partial responsibility to pay a fixed-amount rent to the lessor on certain conditions. It shows that the entrepreneur-lessee can augment his wealth by increasing the risk of the leased firm, as long as his ex-post behavior cannot be detected by the market before signing the contract. Further, the entrepreneur-lessee has no incentive to reinvest his earnings in the leased firm if the rent of the renewed lease contract for the following period is uncertain. These two aspects may have some important long term impacts on the economic development of rural industry.
\end{abstract}

* Dr Hua Yu est professeur de finance à l'Université du Québec à Trois-Rivières. Ses intérêts de recherche incluent le financement des PME, la gestion financière des institutions financières et le financement de projet. Adresse : Département d'administration et d'économique, Université du Québec à Trois-Rivières, 3351, boul. des Forges, Trois-Rivières (Québec) G9A 5H7. 


\section{RESUMEN}

Este artículo analiza la naturaleza y los impactos del contrato de alquiler de las pequeñas empresas chinas en un contexto de sistema de responsabilidad. En un contrato típico, se encuentra, por un lado, el propietario del bien y, por otro, el arrendatario-gestionario, que no asume más que una responsabilidad parcial : la de pagar una cantidad fija al propietario bajo ciertas condiciones. El artículo muestra igualmente que el empresario-arrendatario puede aumentar su riqueza en proporción al riesgo total de la empresa alquilada cuando la información sobre las decisiones exteriores no es divulgada en elmercado. Por otra parte, el empresario-arrendatario no es incitado a reinvertir sus beneficios en la mepresa, teniendo en cuenta la incertidumbre que rodea el beneficio objetivo indicado en el contrato renovado. Estos dos aspectos pueden tener repercusiones importantes en el desarrollo de las empresas rurales a largo plazo.

\section{Introduction}

Au cours des dernières années de la réforme économique chinoise, le monde entier a observé d'importants changements structurels dans les régions rurales de ce pays. Premièrement, les firmes collectives, existant depuis longtemps en Chine, ont évolué graduellement vers un système de propriété quasi privée. Dans ce système appelé «le système de responsabilité », les paysans regroupés en unités familiales disposent du contrôle effectif des terrains et organisent indépendamment leurs activités d'exploitation. Deuxièmement, on a assisté à un développement rapide des entreprises rurales, grâce à une diminution des restrictions et du contrôle gouvernemental. Ces développements n'ont pas été observés uniquement dans le secteur agricole, mais ont touché presque tous les secteurs d'activité économique. Le développement de ces entreprises a eu des conséquences très favorables pour l'économie sur les plans national et régional : il a créé de nouveaux emplois et a augmenté les revenus des habitants ruraux (Wong, 1987 ; Zhu, 1990).

Dans le développement de l'ensemble des entreprises rurales, l'évolution a amené la séparation du droit de propriété et du contrôle surtout dans les entreprises villageoises. Selon ce système de responsabilité qui s'étend maintenant aux entreprises rurales, un particulier peut louer, par soumission, une entreprise appartenant normalement aux autorités gouvernementales (en chinois, on appelle ce système Chengbao). Il contrôle cette entreprise durant la période précisée au contrat, et s'engage à payer un montant fixe au propriétaire-locateur à la fin. 
L'introduction du système de responsabilité dans les entreprises rurales a contribué à une amélioration sensible de son efficacité, ainsi qu'au développement rapide d'une classe d'entrepreneurs-gestionnaires. Cependant, la performance de ce secteur affiche certaines faiblesses, notamment en ce qui concerne les politiques d'investissement et de développement à long terme: d'une part, on observe un faible ratio de réinvestissement des bénéfices nets et, d'autre part, on constate que la plupart des projets d'investissement adoptés sont de courte durée avec des rendements élevés mais volatiles (Byrd et Lin, 1990).

Le but de la présente étude est d'analyser la décision d'investissement des petites entreprises chinoises fonctionnant dans un système de responsabilité. On y examine la décision d'investissement dans un contexte de marché imparfait et le problème de risque moral généré par le contrat, dans un tel système.

La théorie classique en sciences économiques ne reconnaît pas le rôle d'entrepreneur dans un marché en équilibre. Cette version est toutefois corrigée par les économistes qui croient à la persistance d'une économie en déséquilibre. Le rôle d'entrepreneur est indispensable lorsque l'information sur le marché n'est pas complète (Hayek, 1949; Kirzner, 1976). Les entrepreneurs, pour quelque raison, possèdent en général plus de connaissances pertinentes sur le marché que les autres agents économiques. Dans un environnement incertain, l'entrepreneur absorbe le risque et réduit les coûts de l'incertitude pour les investisseurs (Knight, 1942).

La théorie d'agence permet d'avoir une meilleure connaissance de la relation entre l'entrepreneur-gestionnaire et le propriétaire-commettant (Jensen et Meckling, 1976; Harris et Raviv, 1979; Fama, 1980; Grossman et Hart, 1983 ; Lambert, 1983). Dans un contexte d'information asymétrique entre le commettant et son agent, les études montrent qu'un contrat sans compatibilité de motivation peut déclencher des problèmes de risque moral et de conflit d'intérêts. Par conséquent, les deux parties au contrat ne peuvent partager le risque de façon efficiente. Certaines études, qui analysent les liens entre la rémunération d'un agent et sa performance, montrent que le problème du risque moral est souvent engendré par la fonction de rémunération (Liu, 1986). Ce problème peut toutefois être éliminé par un programme de rémunération adéquat (Nalebuff et Stiglitz, 1983; Herriet et Rochet, 1986; Osbend, 1987).

Bien que le problème du risque moral fasse l'objet de débats dans la littérature des sciences économiques et administratives, l'analyse du contrat de location des entreprises exploitées dans un système de responsabilité est une contribution originale. Il s'agit, en fait, d'un phénomène récent et unique qui a pris de l'importance à la suite de réforme économique entreprise dernièrement en Chine. Cette réforme va probablement transformer le système actuel de planification en un système de marché. 
Le contexte chinois renferme certaines particularités intéressantes. D'une part, la relation commettant-agent, souvent décrite dans la littérature, est remplacée par celle de locataire-locateur. D'autre part, les recherches publiées à ce jour ont essentiellement porté sur la relation entre les gestionnaires et les actionnaires de grandes entreprises, alors que la présente étude traite des problèmes spécifiques aux petites entreprises rurales, exploitées dans un pays en voie de développement. Finalement, les entrepreneurs-locataires de ces petites entreprises rurales chinoises sont des spécialistes qui ne possèdent pas de capitaux, mais qui ont des connaissances particulières. Ils ont le contrôle des entreprises en tant que gestionnaires, mais ils sont rémunérés comme des actionnaires en recevant une partie importante du bénéfice net, sans toutefois détenir de titres de propriété. Ce statut unique n'a pas encore été traité dans la littérature.

Dans cet article, nous allons d'abord analyser les caractéristiques particulières des entreprises rurales chinoises (section 1) pour ensuite développer un modèle qui permette d'analyser le choix de projet d'investissement et le lien entre le problème de risque moral et la responsabilité partielle (section 2 ). Nous examinerons ensuite de quelle façon un entrepreneur détermine le montant du revenu réinvesti dans son entreprise, et nous traiterons de l'impact de l'incertitude du revenu cible et du contenu du contrat sur la décision d'investissement (section 3).

\section{Les entreprises rurales et le système de responsabilité}

La location d'une entreprise, auparavant contrôlée par le gouvernement, à un entrepreneur particulier est vue comme une solution de rechange à la privatisation (Cheung, 1989). Il y a plus de quarante ans, la révolution chinoise a remplacé l'économie privée par une économie socialiste. Présentement, on assiste à un renversement de la situation par rapport à la tendance des années 50 .

L'économie centralisée avec les propriétés d'État et de quasi-État (collective) avait montré, d'une part, qu'elle pouvait permettre à une immense population d'assurer sa survie et, d'autre part, qu'elle ne pouvait utiliser efficacement les ressources pour le développement économique à long terme. L'une des solutions qui permettent d'améliorer l'efficacité dans la production, tout en gardant le système de propriété du pays socialiste, est le système de responsabilité. Dans ce système, la décision d'exploitation est basée sur les principes de l'économie de marché. Cependant, l'État demeure le propriétaire légal des ressources. Selon Friedman (1989), le système de responsabilité permet de transformer graduellement une économie de planification centrale vers une économie de marché, sans nécessairement provoquer des chocs économiques et sociaux. 
L'introduction du système de responsabilité est essentielle à la réforme économique dans le secteur rural. Avant la réforme, il existait deux catégories d'entreprises: les entreprises de la commune et les entreprises de brigade de production (village). Les deux catégories d'entreprises étaient effectivement contrôlées par les autorités de deux niveaux respectifs : l'autorité de la commune et celle de la brigade de production. La réforme économique a amené l'émergence d'une troisième catégorie d'entreprises, soit les entreprises privées. Le tableau 1 illustre l'évolution de la structure des entreprises durant la période s'étendant entre 1980 et 1986.

\section{TABLEAU 1}

Répartition de la production annuelle, selon trois catégories d'entreprises rurales (en \%) ${ }^{1}$

\begin{tabular}{|l|c|c|c|c|c|}
\hline Catégories d'entreprises & $\mathbf{1 9 8 0}$ & $\mathbf{1 9 8 3}$ & $\mathbf{1 9 8 4}$ & $\mathbf{1 9 8 5}$ & $\mathbf{1 9 8 6}$ \\
\hline Entreprises de commune & 52,8 & 54,5 & 50,5 & 45,1 & 43,5 \\
\hline Entreprises de village & 41,8 & 37,1 & 39,6 & 37,9 & 35,2 \\
\hline Entreprises privées & 5,4 & 8,4 & 9,9 & 17,0 & 21,3 \\
\hline Total des entreprises rurales $^{2}$ & 100,0 & 100,0 & 100,0 & 100,0 & 100,0 \\
\hline
\end{tabular}

1. Source: Bureau Statistiques d'État Chinois.

2. La catégorie des entreprises privées comprend des entreprises individuelles et des entreprises de partenaires.

La proportion d'entreprises privées a augmenté de 5,4\% à 21,3\% en 6 ans. Cette augmentation s'est faite aux dépens des entreprises de commune et de village. Parmi les trois groupes, c'est celui des entreprises privées qui a eu le taux de croissance le plus élevé. Pour la période de 1980-1986, le taux de croissance annuel de production industrielle du groupe des entreprises privées était de $60,5 \%$ tandis que celui de l'ensemble des entreprises rurales durant la même période n'était que de $27,9 \%$.

Ces quelques données statistiques sous-estiment toutefois la proportion d'entreprises privées dans le total des entreprises rurales. Dans les deux premières catégories d'entreprises, surtout parmi les entreprises de village, plusieurs sont louées aux entrepreneurs professionnels. Ces entreprises ont effectivement un caractère privé selon la nature du contrôle et la distribution du bénéfice. Le contrat de location d'entreprise mène à une séparation du contrôle et du droit légal de propriété ; il transfère à l'entrepreneur-locataire le droit d'utiliser les ressources et de réclamer le revenu résiduel du propriétaire. Malgré le fait que le village garde toujours son droit légal de propriété, l'entrepreneur-locataire est le contrôleur effectif. La différence entre une entreprise louée et une entreprise 
privée est que l'entrepreneur de la première n'a pas le titre légal du droit du propriétaire.

Il est évident que sous le système de responsabilité, les autorités perdent le contrôle sur les entreprises louées. De plus, ils ne reçoivent qu'une partie du revenu des entreprises. Trois motifs ont pu amener les autorités à introduire ce système de responsabilité

1. Le besoin urgent d'entrepreneurs pour activer l'économie.

2. Les ressources financières engendrées par ces entreprises.

3. La nécessité de concilier les intérêts d'un système d'économie socialiste et ceux d'une économie de marché.

\subsection{Le besoin urgent d'entrepreneurs pour activer l'économie}

L'expérience passée a monté que la performance des entreprises contrôlées par le gouvernement était plutôt faible. La bureaucratie étatique détruit l'efficacité des entreprises. Dans l'ancien système, les responsables des entreprises étaient souvent choisis selon des critères autres que leur compétence de gestionnaire. Afin d'améliorer l'efficacité des entreprises rurales, les autorités doivent maintenant engager des entrepreneurs compétents. Avant la révolution de 1949, l'économie chinoise était fondée largement sur les petites économies rurales de type familial ; les échanges commerciaux comptaient parmi les activités les plus importantes dans les régions. Même après la révolution de 1949, l'autorité a permis l'utilisation de certains éléments de l'économie privée, surtout dans les régions rurales. Par rapport à l'économie collective, cette économie privée est toutefois demeurée très marginale. Dans les communes, il existait des petits marchés où les paysans pouvaient vendre leurs produits, ce qui leur a permis de demeurer en contact avec les rudiments du commerce.

L'introduction du système de responsabilité dans le secteur agricole en 1979 a permis d'augmenter l'importance relative de cette économie de marché, et elle devrait favoriser l'émergence d'une classe d'entrepreneurs régionaux. Les gestionnaires des petites entreprises rurales possèdent une meilleure connaissance du fonctionnement de l'économie de marché que leurs homologues des grandes entreprises. Avant l'introduction du système de responsabilité dans les entreprises, l'économie chinoise possédait trois différents niveaux structurés de planification industrielle soit: central, provincial et régional (voir la figure 1). À ces niveaux de planification, ce sont les gouvernements central, provinciaux et régionaux qui régissent le commerce, fixent les prix des biens et contrôlent tout le fonctionnement des entreprises de l'État central, des provinces et des régions: formation de la main-d'œuvre, orientation des marchés, choix des 
fournisseurs. Les deux derniers niveaux d'autorité, soit de commune et de village, ne possèdent pas de planification aussi structurée.

FIGURE 1

Système de planification industrielle de l'économie chinoise

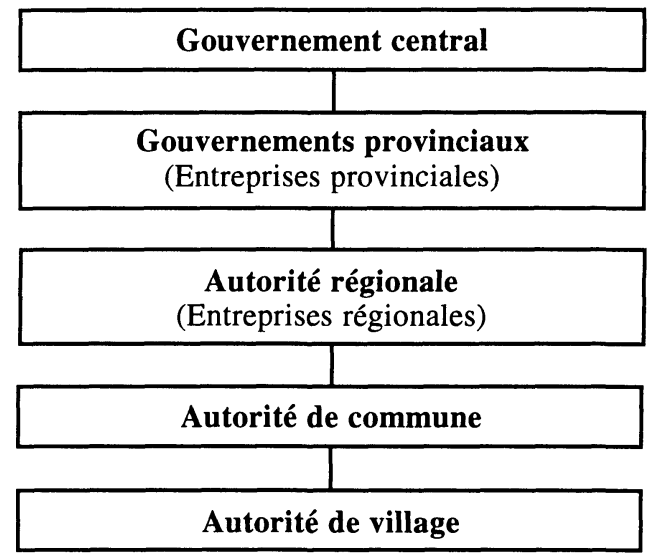

Ce système de planification a obligé les entrepreneurs des entreprises contrôlées par les autorités de commune et de village à organiser l'exploitation, à trouver des clients pour leurs produits et à développer des plans stratégiques dans un univers incertain, etc. Par conséquent, les gestionnaires de ces entreprises constituent maintenant les ressources humaines les plus importantes et sont indispensables au bon fonctionnement du système de responsabilité implanté dans les entreprises rurales.

\subsection{Les ressources financières engendrées par ces entreprises}

Les niveaux hiérarchiques les plus bas de l'autorité rurale, soit les autorités de commune et de village, ont perdu graduellement leur pouvoir et leur rôle, avec l'évolution du système de responsabilité dans le secteur agricole. Étant donné que l'exploitation est désormais organisée par les paysans indépendants, les gouvernements central et provinciaux ont décidé d'abolir le niveau d'autorité de village et de limiter le pouvoir et les budgets accordés à l'autorité de commune. Les revenus des entreprises de commune deviennent une ressource financière essentielle à la commune : ils permettent de financer le développement de programmes sociaux et économiques. Pour augmenter ses ressources financières, l'autorité de commune préfère louer certaines des entreprises les moins rentables, surtout des entreprises de commune, à des personnes intéressées 
et compétentes qui pourront ainsi leur verser un loyer fixe. Certaines des entreprises de village sont privatisées alors que d'autres sont louées à des gestionnaires ; le reste est pris en charge par les paysans des villages.

\subsection{La nécessité de concilier les intérêts d'un système d'économie socialiste et ceux d'une économie de marché}

La location d'une entreprise publique à un entrepreneur particulier est la meilleure façon de résoudre les conflits fondamentaux entre le système socialiste et le mécanisme du système économique de marché. Elle permet aux autorités de garder leur droit légal sur les propriétés des entreprises et en même temps do profiter de l'efficacité de l'économie de marché.

\section{Responsabilité partielle, risque moral et choix d'investissement}

Dans cette section, nous analysons, dans un état statique, le contrat de location d'une entreprise rurale. Les deux parties qui s'engagent dans le contrat, le propriétaire-locateur et l'entrepreneur-locataire, veulent optimiser leurs objectifs pour une période donnée. Le modèle que nous utilisons permet d'abord une analyse de la décision d'investissement en contexte de contrat parfait. Par la suite, nous introduisons dans le modèle les éléments imparfaits du contrat de location du système de responsabilité afin d'examiner l'impact du risque moral sur le choix des projets d'investissement.

Selon le contrat de location, le gestionnaire-locataire s'engage à payer un montant fixe au propriétaire-locateur vers la fin du contrat, si le bénéfice net est supérieur à ce montant. De plus, le locataire peut garder une portion du montant du bénéfice excédentaire et remettre le reste au locateur, et même dans certains cas, le locataire peut conserver la totalité du montant excédentaire. Durant la période stipulée dans le contrat, le gestionnaire-locataire prend le contrôle de l'entreprise. Le choix du locateur se fait généralement selon les méthodes suivantes: l'appel d'offre; l'élection par les employés; la recommandation de l'autorité, confirmée par l'assemblée des employées.

Un contrat de location d'entreprise, dans le contexte du système de responsabilité chinoise, est différent d'un contrat de location nord-américain, à cause du droit du locateur sur l'entreprise louée. Le locateur chinois est le propriétaire de l'entreprise. Il ne reçoit son bénéfice qu'après le remboursement de la dette et le paiement des intérêts, ce qui l'amène à subir le risque financier total de l'entreprise. Par contre, en Amérique du Nord, le locateur d'un bien a un droit prioritaire de recevoir le loyer; il est propriétaire de l'actif loué mais créancier 
de l'entreprise. Le contrat nord-américain est donc moins risqué, pour le locateur, qu'un contrat signé dans un système de responsabilité.

Le contrat de location des entreprises séparant le contrôle et la propriété peut provoquer un conflit d'intérêts entre l'entrepreneur-locataire et le propriétaire-locateur. Selon le contrat, les rendements et les risques courus par les deux parties sont différents. Le locateur a la priorité de recevoir le revenu cible comme loyer. La responsabilité de l'entrepreneur-locataire est toutefois partielle: son gain est zéro si le bénéfice de l'entreprise est plus petit que le bénéfice cible, et il augmente en fonction du bénéfice résiduel une fois le loyer payé. Cette distribution asymétrique de la valeur nette est à l'origine du conflit d'intérêts entre les deux parties quant à la décision d'investissement dans l'entreprise. L'entrepreneur de l'entreprise contractée choisirait les projets dont les niveaux de risque et de rendement particuliers maximisent sa propre richesse.

Dans la présente analyse, le propriétaire-locateur est supposé rationnel. Il cherche un contrat lui permettant de recevoir un montant maximal de revenu ajusté au risque. Son objectif est de maximiser l'utilité de sa richesse, exprimée par la fonction $U$. Cette fonction est concave au niveau de la richesse tel que $U^{\prime}>0, U^{\prime \prime}<0$. Le rendement éventuel de l'entreprise à la fin d'une période donnée est une variable stochastique, $Y(\theta)$, où q est un indicateur de risque. La fonction de densité du rendement est $f(Y)$. Le gain du propriétaire-locateur dépend du bénéfice cible de l'entreprise, $Y^{*}$. Quand $Y>Y^{*}$, l'entrepreneur paie $Y^{*}$ au propriétaire-locateur, sinon le propriétaire reçoit le bénéfice de l'entreprise, $Y$, lequel pourrait être négatif.

Le gain de l'entrepreneur-locataire est une portion, $w<1$, du montant résiduel de l'entreprise. Plus précisément, son revenu est $W=W\left(Y-Y^{*}\right)$ si $Y>Y^{*}$, ou zéro si $Y<Y^{*}$. On considère que l'entrepreneur-locataire a une aversion pour le risque. Sa fonction d'utilité est égale à $E\left[W\left(Y^{*}, \theta\right)\right]-C\left(Y^{*}\right)$ $+V(\theta) . C\left(Y^{*}\right)$ est le coût de renonciation qui augmente avec le bénéfice cible. D'autre part, $V(\theta)$ mesure l'aversion de l'entrepreneur face au risque; naturellement, $\mathrm{V}^{\prime}(\theta)<0, \mathrm{~V}^{\prime \prime}(\theta)<0$.

D'abord, considérons une situation dans laquelle l'entrepreneur-locataire est incapable de changer le niveau du risque de l'entreprise. Le propriétaire veut choisir un niveau de bénéfice cible qui maximise son objectif. Normalement, le revenu du propriétaire augmente avec le niveau du bénéfice cible, mais si celuici est trop élevé, le contrat peut devenir inacceptable pour l'entrepreneur. La fonction objectif dans l'équation (1) ci-dessous décrit la décision du propriétaire.

$\operatorname{Max}$

$$
Z=E\left[U\left(Y-W \max \left(Y-Y^{*}, 0\right)\right]\right.
$$


soumise à la contrainte suivante :

$E\left[W\left(Y^{*}, \theta\right)\right]-C\left(Y^{*}\right)+V(\theta)>0$

où

$\infty$

$E\left[W\left(Y^{*}, \theta\right)\right]=W \int\left(Y-Y^{*}\right) f(Y, \theta) d Y$

$$
Y^{*}
$$

Le contrat est acceptable pour l'entrepreneur-locataire lorsque $E\left[W\left(Y^{*}, \theta\right)\right]-C\left(Y^{*}\right)+V(\theta)>0$, c'est-à-dire que le revenu espéré ajusté au risque doit être positif. Le propriétaire-locateur choisit un niveau particulier de $Y^{*}$ qui maximise son utilité espérée. Le problème d'optimisation se présente par la fonction de Lagrange ci-dessous :

$\infty$

$$
\begin{gathered}
L=\int U(Y) f(Y, \theta) d Y-\int U\left[W\left(Y-Y^{*}\right)\right] f(Y,(\theta) d Y \\
Y^{*} \\
+\lambda_{1}\left[W \int\left(Y-Y^{*}\right) f(Y, \theta) d Y-C\left(Y^{*}\right)+V(\theta)\right] \\
Y^{*}
\end{gathered}
$$

En utilisant le calcul intégral, le revenu espéré de l'entrepreneur peut se réécrire de la façon suivante:

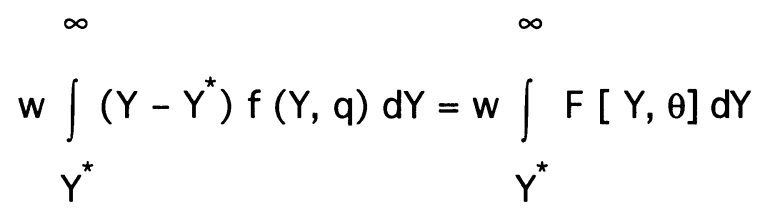

La condition d'optimisation de l'équation (4), en utilisant l'équation (5), exige que l'équation suivante soit satisfaite:

$\frac{U^{\prime} w F\left[Y^{\star}\right]}{w F\left[Y^{\star}\right]-C^{\prime}\left(Y^{\star}\right)}=\lambda$

$\forall \mathrm{Y}$ 
Le résultat de l'équation (6) semble indiquer un partage de risque optimal du type Pareto. Le paramètre de risque, $\theta$, est déterminé avant la négociation ảu contrat de location. L'entrepreneur-locataire ne peut changer le niveau de risque des projets d'investissement. L'équation (6) est donc une situation de coopération où les deux parties du contrat maximisent leurs objectifs simultanément.

Cependant, si le contrat permet au locataire de changer le niveau du risque de l'entreprise, la solution d'optimisation est différente: il ne choisit que des projets qui lui permettent d'obtenir le revenu espéré le plus élevé. Pour sa part, la maximisation du bénéfice personnel requiert la condition suivante:

$\infty$

$\int F \theta\left[Y^{*}\right] d Y+V^{\prime}(\theta)=0$

$Y^{*}$

Le bénéfice espéré marginal de l'entrepreneur-locataire est égal au coût marginal lié à l'augmentation du risque des projets d'investissement. Compte tenu que l'entrepreneur-locataire contrôle la variable $\theta$, cette condition est constamment maintenue. Par conséquent, la fonction d'optimisation du propriétaire-locateur est modifiée:

$$
\underset{Y^{*}}{\operatorname{Max}} E[U(Y-W)]=E\left[U\left(Y-W \max \left(Y-Y^{*}\right)\right)\right]
$$

et soumise aux contraintes suivantes:

$\infty$

$W$
$Y^{*}$

$\infty$

$\int F_{\theta}\left[Y^{*}\right] d Y+V^{\prime}(\theta)=0$

La nouvelle équation de Lagrange avec les deux contraintes ((9) et (10)) devient maintenant : 


$$
\begin{aligned}
& L=\int U(Y) f(Y, \theta) d Y-\int_{Y^{*}}^{\infty} U\left[W\left(Y-Y^{*}\right)\right] f(Y, \theta) d Y \\
& +\lambda_{1}\left[W \int_{Y^{*}}^{\infty}\left(Y-Y^{*}\right) f(Y, \theta) d Y-C\left(Y^{*}\right)+V(\theta)\right] \\
& \infty \\
& +\lambda_{2}\left[W \int_{Y^{*}}^{\infty}\left(Y-Y^{*}\right) \cdot f_{\theta}(Y, \theta) d Y+V^{\prime}(\theta)\right]
\end{aligned}
$$

Les solutions d'optimisation s'écrivent comme suit :

$$
\frac{U^{\prime} W F\left[Y^{*}\right]}{w F\left[Y^{*}\right]-C^{\prime}\left(Y^{*}\right)}=\lambda_{1}+\lambda_{2} \frac{w F \theta\left[Y^{*}\right]}{w F\left[Y^{*}\right]-C^{\prime}\left(Y^{*}\right)} \quad \forall Y
$$

La valeur de $\lambda_{2}$ est positive selon la condition d'égalité. Les résultats de l'équation (12) nous indiquent que lorsque l'entrepreneur-locataire contrôle le risque du projet, le propriétaire-locateur a tendance à choisir un $Y^{*}$ moins élevé étant donné son coût marginal supérieur. D'autre part, le résultat de l'équation (7) indique que le niveau de risque de l'investissement est plus élevé que celui avec un contrat parfait. Ceci augmente le gain espéré de l'entrepreneur, tandis que le revenu du propriétaire-locateur diminue et devient plus risqué. Par conséquent, le partage du risque entre les deux parties ne peut être optimal ; il amène éventuellement un équilibre sous-optimal par rapport à celui de Pareto.

Le problème est dû à la responsabilité partielle de l'entrepreneur. Comme son revenu est lié à la rentabilité de l'entreprise, il n'a pas de salaire fixe et n'a rien à perdre si le bénéfice réalisé est inférieur au bénéfice cible. Par contre, lorsque le rendement dépasse le niveau cible, il reçoit un gain. Cette distribution asymétrique l'encourage donc à prendre plus de risques. Un projet plus risqué augmente l'étendue de la distribution des bénéfices, et par conséquent, les probabilités d'atteindre le niveau cible sont plus élevées également. Cependant, l'augmentation du gain espéré de l'entrepreneur-locataire réduit le rendement espéré du propriétaire et son utilité, car les projets choisis par l'entrepreneur-locataire sont plus risqués. Malheureusement, le contrat ne donne pas de protection au propriétaire-locateur, et il ne peut plus intervenir sur la décision de projets d'investissement durant la période du contrat. 


\section{Bénéfice cible incertain et motif de sous-investissement}

Le modèle de la section précédente nous a permis d'analyser le choix de projet d'investissement d'une entreprise évoluant dans un système de responsabilité, étant donné le montant de l'investissement. Dans la présente section nous examinons, avec ce modèle, la détermination du montant optimal d'investissement.

La théorie néo-classique suggère la séparation de la décision d'investissement de celle de financement dans un marché parfait où les investisseurs peuvent obtenir autant de financement qu'ils le désirent. Cependant, dans un marché qui subit beaucoup de restrictions comme c'est le cas en Chine, les entreprises rurales font face à des contraintes financières très importantes. D'une part, le financement personnel accordé par les institutions financières n'existe pratiquement pas, la consommation des ménages dépend donc entièrement de leur revenu. Il n'y a à toutes fins utiles pas de marchés des capitaux pour les investissements privés, cela signifie que les décisions de financement et d'investissement sont indissociables. D'autre part, le financement externe est généralement difficile à obtenir pour les entreprises rurales: celles-ci doivent donc s'autofinancer. Ceci rend le rôle de l'entrepreneur extrêmement important dans la décision d'investissement, d'autant plus qu'en prenant une telle décision, il a le contrôle effectif de l'entreprise. De plus, il a les moyens d'investir puisqu'il reçoit une bonne partie du rendement de l'entreprise, la seule source de financement disponible.

Dans la présente analyse, l'entrepreneur est le locataire actuel de l'entreprise. La décision d'investissement se fait avant l'échéance du contrat de location; cet investissement ne changera pas le flux monétaire de la période courante, mais il aura un impact sur le rendement de la prochaine période. (Nous utilisons les mêmes symboles que dans la section précédente). Considérons $Y$ comme étant le rendement de la prochaine période. Il est fonction de deux variables, le risque $\theta$ et le montant d'investissement $I$.

L'entrepreneur-locataire prend la décision d'investissement et maximise la fonction suivante:

$$
\operatorname{Max} Z=E\left[W\left(Y-Y^{*}, \theta, I\right)\right]-C\left(Y^{*}, I\right)+V(\theta, I)
$$

Pour que cet investissement soit rentable, le revenu généré par le nouvel investissement devrait être supérieur au coût de renonciation de l'investissement, $C\left(Y^{*}, I\right)$. Ce coût représente la préférence de consommation intertemporelle de l'investisseur, (en l'occurence l'entrepreneur). Le coût total augmente avec le montant de l'investissement, $C^{\prime}(I)>0$. De plus, la valeur de l'investissement doit être ajustée au risque, ceci signifie donc que $V^{\prime}(I)<0$. 
L'optimisation de l'équation (13) par rapport à I donne :

$\infty$

$w \int_{Y^{*}} F_{1}[Y] d Y-C^{\prime}(I)+V^{\prime}(I)=0 \quad \forall Y$

Le rendement espéré marginal de l'investissement doit être égal au coût marginal ajusté au risque. Le résultat de l'équation (14) est la décision d'investissement dans un marché parfait. Le contrat imparfait de location d'entreprise sous le système de responsabilité exige donc certaines modifications de cette condition.

Le contrat est normalement valide pour une période limitée; à l'échéance, les deux parties peuvent le renégocier et le renouveler. Comme le bénéfice cible du nouveau contrat peut être différent du contrat précédent, la cible à long terme est une variable incertaine. L'autre aspect imparfait du contrat est que la valeur de l'actif de l'entreprise n'est pas divisible entre l'entrepreneur-locataire et le propriétaire-locateur, ce dernier maintenant son droit de propriété sur l'actif de l'entreprise. L'entrepreneur n'a pas de titre de propriétaire de l'actif de l'entreprise dans laquelle il avait investi. Par contre, le propriétaire-locateur peut réclamer son droit sur la valeur investie par l'entrepreneur-locataire durant la période du contrat. La vie effective d'un projet est souvent plus longue que la durée du contrat de location. Si l'entrepreneur-locataire décide de ne pas renouveler le contrat, il perd la valeur résiduelle de l'investissement.

L'analyse comparative statique ci-dessous montre l'effet de l'incertitude du bénéfice cible sur la décision d'investissement de l'entrepreneur-locataire:

$$
\frac{\partial^{2} Z}{\partial \partial Y^{*}}=w F_{I}\left[Y^{*}\right]=<0
$$

Lorsque le propriétaire-locateur augmente le bénéfice cible de la prochaine période, le rendement marginal de l'investissement de l'entrepreneur-locataire diminue. Cette augmentation du niveau cible permet au propriétaire-locateur de profiter d'une partie du rendement du nouvel investissement. Le bénéfice réinvesti par l'entrepreneur sert de nantissement l'obligeant à accepter cette augmentation du bénéfice cible, étant donné que cette augmentation est moins élevée que le rendement total de l'investissement. L'entrepreneur connaît l'intention du propriétaire-locateur d'augmenter le revenu cible dans le prochain contrat s'il investit et prévoit la possibilité de diminution de son rendement 
marginal espéré. Il réduit, par conséquent, le montant optimal du bénéfice réinvesti tout en augmentant le niveau de sa consommation.

\section{Conclusion}

Cette étude analyse les contrats de location des entreprises rurales sous le système de responsabilité en Chine. On y traite deux aspects particuliers : la tendance de l'augmentation du risque des entreprises contractées et le problème de sous-investissement. Ces deux problèmes sont effectivement liés au contrat imparfait de location.

Le contrat exige la responsabilité partielle de la part de l'entrepreneur. Ce dernier peut recevoir une proportion du bénéfice résiduel si l'entreprise réalise un revenu supérieur au bénéfice cible payé au propriétaire-locateur. Cependant, l'entrepreneur n'a aucune obligation lorsque le bénéfice de l'entreprise est en deçà du niveau cible. Dans tous les cas, il obtient un gain non négatif et son gain espéré augmente avec le niveau du risque de l'entreprise. Puisque le contrat de location lui permet de contrôler la décision d'investissement, il a tendance à augmenter le risque de l'entreprise.

Le contrat d'une durée fixe génère de l'incertitude quant au bénéfice cible à long terme. À l'échéance du contrat, le propriétaire a la possibilité d'augmenter le bénéfice cible pour la période suivante. Le bénéfice réinvesti de l'entrepreneur pendant la période précédente sert de nantissement qui l'oblige à accepter l'augmentation du bénéfice cible, puisque le contrat ne donne aucune protection sur le nouvel investissement de l'entrepreneur. Le non-renouvellement du contrat permet au propriétaire d'exercer son droit de propriété sur l'ensemble de la valeur de l'entreprise, y compris le nouvel investissement. Cette situation décourage l'entrepreneur à investir dans l'entreprise, puisqu'il risque de perdre les sommes qu'il y aura injectées.

L'introduction du système de responsabilité dans les entreprises rurales a contribué énormément au développement économique régional. Cependant, les aspects imparfaits du contrat de location d'entreprises ont des impacts négatifs sur l'investissement des entreprises louées et le développement économique à long terme. Pour réduire l'effet du risque moral de la part de l'entrepreneur, le contrat doit être modifié de façon à ce que l'entrepreneur soit responsable de tout état d'exploitation de l'entreprise. Dans le cas où le rendement serait inférieur au niveau cible, l'introduction d'une pénalité sur le revenu de l'entrepreneur serait nécessaire, permettant ainsi de décourager l'entrepreneur d'adopter des projets comportant un risque élevé. Dans ce cas, une condition serait essentielle: au moins une partie du revenu total de l'entrepreneur devrait être fixe. 
Afin de réduire le conflit d'intérêts causé par l'ambiguiité du droit de propriété, la valeur de l'entreprise doit être divisible entre le propriétairelocateur et l'entrepreneur-locataire. Un modèle de société anonyme ou de partenariat entre les deux parties pourrait permettre de définir plus clairement les droits de propriété. Les solutions concrètes des problèmes de risque moral et de conflit d'intérêts feront l'objet d'une prochaine étude.

\section{Bibliographie}

BYRD, W.A. (1990), « Entrepreneurship, capital and ownership » dans W.A. Byrd et Q. Lin (éditeurs), China's Rural Industry, Oxford University Press.

BYRD, W.A. et Q. Lin (1990), « China's rural industry : an introduction » dans W.A. Byrd et Q. Lin (éditeurs), China's Rural Industry, New York, Oxford University Press.

CASSON, M. (1991) «L'entrepreneur », Paris, Économica.

Cheung, S.N. (1989), « Privatization vs. special interests : the experience of China's economic reforms » dans James A. Dorn et X. Wang (éditeurs), Economic Reform in China. Problems and Prospects, Chicago, The University of Chicago Press.

FAMA, E. (1980), «Agency problem and the theory of the firm », Journal of Political Economy, vol. 88, n. 2, p. 288-307.

FRIEDMAN, M. (1989), Using the market for social development dans James, A. Dorn et X. Wang, Opus Cité.

GROSSMAN, S.J. et O.D. HART (1983), « An analysis of the principal-agent problem » Econometrica, vol. 51, p. 7-45.

HAYEK, F.A. (1949), «The meaning of competition » dans F.A. Hayek (éditeur), Individualism and Economic Order, Routledge and Kegan Paul, Londres, p. 92106.

HARRIS, M. et A. RAVIV (1979), « Optimal incentive contracts with imperfect information », Journal of Economic Theory, vol. 20, p. 231-259.

HenRIET, D. et J.C. RoCHET (1986), « La logique des systèmes bonus-malus en assurance automobile : une approche théorique », Annales d'Économie et de Statistique, vol. 1, p. 133-152.

HolmstRöM, B. (1979), « Moral hasard and observability », Bell Journal of Economics, vol. 10, p. 74-91. 
JENSEN, M. et W. MECKLING (1976), « Theory of firm : managerial behavior, agence costs and ownership structure », Journal of Financial Economics, octobre, p. 305-360.

KIRZNER, I.M. (1976), «Equilibrium Versus Market Process » dans E.G. Dolan (éditeur), The Foundations of Modern Austrian Economics, Kansas City, Sheet and Word, p. 115-125.

KNIGHT, F.H. (1942), « Profit and entrepreneurial function», The Task of Economic History : Supplement to Journal of Economic History, vol. 2, p. 126-132.

LAMBART, R. (1983), "Long term contracts and moral hasard », Bell Journal of Economics, vol. 14, p. 441-452.

LIU, P.W. (1986), « Moral hasard and incentives in decentralized planning environment », Journal of Comparative Economics, vol. 10, p. 91-105.

NalebufF, B. et J. Stiglitz (1983), « Prizes and incentives : towards a general theory of compensation and competition », Bell Journal of Economics, vol. 14, p. 21-43.

OSBEND, B. (1987), « Speak softly, but carry a big stick : on optimal targets under moral hasard », Journal of Comparative Economics, vol. 11, p. 584-595.

WONG, C.P. (1987), « Between plan and market : the role of the local sector in post-Mao China », Journal of Comparative Economics, vol. 11, p. 385-398.

ZhU, L. (1990), Rural Reform and Peasant Income in China: The Impact of China's Post-Mao Rural Reforms in Selected Regions, New York, St. Martin's Press. 